S. Kaneyuki and F. L. Williams

Nagoya Math. J.

Vol. 99 (1985), 173-187

\title{
ALMOST PARACONTACT AND PARAHODGE STRUCTURES ON MANIFOLDS
}

\author{
SOJI KANEYUKI AND FLOYD L. WILLIAMS
}

\section{§. Introduction}

In this paper we study the paracomplex analogues of almost contact structures, and we introduce and study the notion of parahodge structures on manifolds. In particular, we construct new examples of paracomplex manifolds and we find all simply connected parahermitian symmetric coset spaces, which are the adjoint orbits of noncompact simple Lie groups, with parahodge structures induced by the Killing forms. This is done by (i) observing that a version of the results of A. Morimoto [4] on almost contact structures can be formulated and proved for almost paracontact structures, and by (ii) the methods of geometric quantization [3] applied to parahermitian symmetric triples [1] in conjunction with results of [7]. Two of the main results are Theorem 2.5 (which ties together the above structures) and Corollary 3.9.

The first author would like to express sincere thanks to the mathematics department of the University of Massachusetts for providing us the pleasant surroundings and facilities to carry out this work.

Notations.

$\boldsymbol{R}^{+}$the group of positive real numbers,

id the identity mapping,

$e$ the unit element of a group,

$G^{0}$ the identity component of a Lie group $G$, $\mathfrak{X}(M)$ the Lie algebra of all smooth vector fields on a manifold $M$.

The Lie algebra of a Lie group $G$ is denoted by the corresponding German small letter $g$ and also by Lie $G$.

\section{§1. Almost paracontact structures}

Definition 1.1. Let $M$ be a $2 n+1$ dimensional smooth manifold. Let Received June 28, 1984. 
$\phi$ be a $(1,1)$-tensor field, $\xi$ a vector field, and $\eta$ a 1-form on $M$. Then the triple $\Sigma=(\phi, \xi, \eta)$ is called an almost paracontact structure on $M$, if the following conditions are satisfied:

(1.2) $\phi^{2} X=X-\eta(X) \xi, X \in \mathfrak{X}(M)$,

(1.3) if we denote by $\{\eta\}^{\perp}$ the subbundle of the tangent bundle $T(M)$ of $M$ consisting of all vectors annihilated by $\eta$, then the tensor field $\phi$ induces an almost paracomplex structure (cf. [1]) on each fibre of $\{\eta\}^{\perp}$.

The pair $(M, \Sigma)$ is called an almost paracontact manifold.

ExAmple 1.2. On the real line $R$ with coordinate $t$, put $\phi=0, \xi=$ $d / d t$ and $\eta=d t$. Then $\Sigma_{0}=(\phi, \xi, \eta)$ is an almost paracontact structure on $\boldsymbol{R}$.

Example 1.3. Let $C$ be the cone $\left\{\left(x_{1}, x_{2}, x_{3}\right) \in R^{3}: x_{1}^{2}+x_{2}^{2}-x_{3}^{2}>0\right\}$. Consider the submanifolds $H_{t}(t>0)$ in $C$ defined by the equation $x_{1}^{2}+$ $x_{2}^{2}-x_{3}^{2}=t$. It is known [1] that $H_{1}$ has a natural paracomplex structure $I_{1}$. Let us consider the vector field and the 1 -form

$$
\begin{aligned}
& \xi=x_{1} \frac{\partial}{\partial x_{1}}+x_{2} \frac{\partial}{\partial x_{2}}+x_{3} \frac{\partial}{\partial x_{3}}, \\
& \eta=\left(x_{1}^{2}+x_{2}^{2}-x_{3}^{2}\right)^{-1}\left(x_{1} d x_{1}+x_{2} d x_{2}-x_{3} d x_{3}\right) .
\end{aligned}
$$

The differential system $\eta=0$ is invariant under the diffeomorphism $\exp t \xi$ on $C$. Therefore, by using the action of $\exp t \xi$, one can extend smoothly $I_{1}$ to the bundle homomorphism $I$ of $\{\eta\}^{\perp}$ with $I^{2}=$ id. The tangent bundle $T(C)$ of $C$ can be written as a whitney sum $T(C)=R \xi \oplus\{\eta\}^{\perp}$. We define the $(1,1)$-tensor field $\phi$ by putting $\phi=0$ on $R \xi$ and $\phi=I$ on $\{\eta\}^{\perp}$. Then it can be verified that $(\phi, \xi, \eta)$ is an almost paracontact structure on $C$.

Proposition 1.4. Let $(M, \Sigma)$ and $(\bar{M}, \bar{\Sigma})$ be two almost paracontact manifolds, where $\Sigma=(\phi, \xi, \eta)$ and $\bar{\Sigma}=(\bar{\phi}, \bar{\xi}, \bar{\eta})$. For $X \in \mathfrak{X}(M), \bar{X} \in \mathfrak{X}(\bar{M})$ let us put

$$
I(X, \bar{X})=(\phi X+\bar{\eta}(\bar{X}) \xi, \bar{\phi} \bar{X}+\eta(X) \bar{\xi}) .
$$

Then $I$ is an almost paracomplex structure on $M \times \bar{M}$.

Proof. By straight-forward computations we have $I^{2}=$ id. We have to show that the \pm 1 -eigenspaces of $I$ are of the same dimension. Let 
$\{\eta\}^{\perp}$ and $\{\bar{\eta}\}^{\perp}$ be the subbundles of $T(M)$ and $T(\bar{M})$ defined by the differential systems $\eta=0$ and $\bar{\eta}=0$, respectively. Then the restrictions of $I$ to $\{\eta\}^{\perp}$ and to $\{\bar{\eta}\}^{\perp}$ are equal to $\phi$ and $\bar{\phi}$, respectively. Furthermore, since we have $I(\xi, \pm \bar{\xi})=( \pm \xi, \bar{\xi}), I$ induces the natural paracomplex structure on $\boldsymbol{R} \xi \oplus \boldsymbol{R} \bar{\xi}$. Consequently, the two eigenspaces of $I$ have the same dimension.

The almost paracomplex structure $I$ above is called the almost paracomplex structure induced by $\Sigma$ and $\bar{\Sigma}$. Now we will give some definitions which are analogous to those in Morimoto [4].

Definition 1.5. Let $(M, \Sigma)$ be an almost paracontact manifold. $\Sigma$ is called integrable if the almost paracomplex structure on $M \times M$ induced by $\Sigma$ and $\Sigma$ is paracomplex.

Definition 1.6. Let $(M, \Sigma)$ be an almost paracontact manifold and let $\left(\boldsymbol{R}, \Sigma_{0}\right)$ be the almost paracontact manifold in Example 1.2. If the almost paracomplex structure on $M \times \boldsymbol{R}$ induced by $\Sigma$ and $\Sigma_{0}$ is paracomplex, then $\Sigma$ is called normal.

Definition 1.7. Let $\Sigma=(\phi, \xi, \eta)$ be an almost paracontact structure on $M$. Let us define a $(1,2)$-tensor field $\psi$ and a 2 -form $\theta$ on $M$ by putting

$$
\begin{aligned}
\psi(X, Y)= & \phi[X, Y]-[\phi X, Y]-[X, \phi Y]+\phi[\phi X, \phi Y] \\
& +(\phi X \cdot \eta(Y)-\phi Y \cdot \eta(X)) \xi, \\
\theta(X, Y)= & \eta([X, Y])-X \cdot \eta(Y)+Y \cdot \eta(X)+\eta([\phi X, \phi Y]),
\end{aligned}
$$

where $X, Y \in \mathfrak{X}(M) . \psi$ is called the torsion tensor field of $\Sigma$.

Theorem 1.8. Let $(M, \Sigma)$ and $(\bar{M}, \bar{\Sigma})$ be almost paracontact manifolds, where $\Sigma=(\phi, \xi, \eta)$ and $\bar{\Sigma}=(\bar{\phi}, \bar{\xi}, \bar{\eta})$. Then the almost paracomplex structure $I$ on $M \times \bar{M}$ induced by $\Sigma$ and $\bar{\Sigma}$ is paracomplex, if and only if the torsion tensor fields $\psi$ and $\bar{\psi}$ of $\Sigma$ and $\bar{\Sigma}$ vanish identically.

Proof. The proof is similar to that for almost contact structures (cf. Morimoto [4]). Let $T$ be the torsion tensor of $I$. Then $I$ is paracomplex if and only if $T$ vanishes identically (cf. [1]). The condition $T=0$ is equivalent to the following

$$
\begin{aligned}
& I[(X, \bar{X}),(Y, \bar{Y})] \\
& \quad=[I(X, \bar{X}),(Y, \bar{Y})]+[(X, \bar{X}), I(Y, \bar{Y})]-I[I(X, \bar{X}), I(Y, \bar{Y})],
\end{aligned}
$$

where $X, Y \in \mathfrak{X}(M)$ and $\bar{X}, \bar{Y} \in \mathfrak{X}(\bar{M})$. This condition is equivalent to the 
following two conditions:

$$
\begin{aligned}
\phi[X, Y] & +\bar{\eta}([\bar{X}, \bar{Y}]) \xi \\
= & {[\phi X+\bar{\eta}(\bar{X}) \xi, Y]-\bar{Y} \cdot \bar{\eta}(\bar{X}) \xi+[X, \phi Y+\bar{\eta}(\bar{Y}) \xi] } \\
& +\bar{X} \cdot \bar{\eta}(\bar{Y}) \xi-\phi[\phi X+\bar{\eta}(\bar{X}) \xi, \phi Y+\bar{\eta}(\bar{Y}) \xi] \\
& -\bar{\eta}([\bar{\phi} \bar{X}+\eta(X) \bar{\xi}, \bar{\phi} \bar{Y}+\eta(Y) \bar{\xi}]) \xi+\bar{f}(X, \bar{X}, Y, \bar{Y}) \xi, \\
\bar{\phi}[\bar{X}, \bar{Y}] & +\eta([X, Y]) \bar{\xi} \\
= & {[\bar{\phi} \bar{X}+\eta(X) \bar{\xi}, \bar{Y}]-Y \cdot \eta(X) \xi+[\bar{X}, \bar{\phi} \bar{Y}+\eta(Y) \xi] } \\
& +X \cdot \eta(Y) \bar{\xi}-\bar{\phi}[\bar{\phi} \bar{X}+\eta(X) \bar{\xi}, \bar{\phi} \bar{Y}+\eta(Y) \bar{\xi}] \\
& -\eta([\phi X+\bar{\eta}(\bar{X}) \xi, \phi Y+\bar{\eta}(\bar{Y}) \xi]) \xi+f(X, \bar{X}, Y, \bar{Y}) \bar{\xi},
\end{aligned}
$$

where we put

$$
\begin{aligned}
& f(X, \bar{X}, Y, \bar{Y}) \\
& \quad=\bar{\phi} \bar{Y} \cdot \bar{\eta}(\bar{X})-\bar{\phi} \bar{X} \cdot \bar{\eta}(\bar{Y})+\eta(Y)(\bar{\xi} \cdot \bar{\eta}(\bar{X}))-\eta(X)(\bar{\xi} \cdot \bar{\eta}(\bar{Y})) \\
& \bar{f}(X, \bar{X}, Y, \bar{Y}) \\
& \quad=\phi Y \cdot \eta(X)-\phi(X) \cdot \eta(Y)+\bar{\eta}(\bar{Y})(\xi \cdot \eta(X))-\bar{\eta}(\bar{X})(\xi \cdot \eta(Y)) .
\end{aligned}
$$

By putting $\bar{X}=\bar{Y}=0$ or $X=Y=0$ in (1.9) and (1.10) we have

$$
\begin{aligned}
& \psi(X, Y)=0, \\
& \theta(X, Y)=0, \\
& \bar{\psi}(\bar{X}, \bar{Y})=0, \\
& \bar{\theta}(\bar{X}, \bar{Y})=0 .
\end{aligned}
$$

By putting $\bar{X}=Y=0$ or $X=\bar{Y}=0$ in (1.9) and (1.10) we have

$$
[X, \bar{\gamma}(\bar{Y}) \xi]-\phi[\phi X, \bar{\eta}(\bar{Y}) \xi]-\bar{\eta}([\eta(X) \bar{\xi}, \bar{\phi} \bar{Y}]) \xi+\bar{\eta}(\bar{Y})(\xi \cdot \eta(X)) \xi=0,
$$

(1.19) $[\bar{\eta}(\bar{X}) \xi, Y]-\phi[\bar{\eta}(\bar{X}) \xi, \phi Y]-\bar{\eta}([\bar{\phi} \bar{X}, \eta(Y) \bar{\xi}]) \xi-\bar{\eta}(\bar{X})(\xi \cdot \eta(Y)) \xi=0$,

$$
[\bar{X}, \eta(Y) \bar{\xi}]-\bar{\phi}[\bar{\phi} \bar{X}, \eta(Y) \bar{\xi}]-\eta([\bar{\eta}(\bar{X}) \xi, \phi Y]) \bar{\xi}+\eta(Y)(\bar{\xi} \cdot \bar{\eta}(\bar{X})) \bar{\xi}=0 .
$$

Note that (1.9) and (1.10) are equivalent to (1.13)-(1.20). By setting $Y=\xi$ in (1.13), we have

$$
\phi[X, \xi]=[\phi X, \xi], \quad X \in \mathfrak{X}(M) .
$$

We have to prove the "if" part of the theorem. This is equivalent to saying that (1.13) and (1.15) imply (1.14), (1.16) and (1.17)-(1.20). This can 
be done in the following way: (1.17) (resp. (1.18)) is equivalent to (1.19) (resp. (1.20)). (1.13) and (1.15) imply (1.17) and (1.20), by using (1.21). On the other hand (1.13) (resp. (1.15)) implies (1.14) (resp. (1.16)). The proof of these facts is quite similar to Morimoto's [4], so we can omit the details.

Corollary 1.9. Let $(M, \Sigma)$ be an almost paracontact manifold. Then $\Sigma$ is integrable if and only if $\psi=0$.

Corollary 1.10. An almost paracontact structure $\Sigma$ is integrable if and only if $\Sigma$ is normal. In particular, $\Sigma$ is normal if and only if $\psi=0$.

We will give an example of almost paracontact structure with $\psi=0$.

Proposition 1.11. The almost paracontact structure $\Sigma=(\phi, \xi, \eta)$ on the cone $C$ given in Example 1.3 satisfies the condition $\psi=0$.

Proof. Since $\psi$ is a tensor field, it is sufficient to show that $\psi(X, Y)=0(X, Y \in \mathfrak{X}(C))$ for the following three cases: (a) $X=Y=\xi$, (b) $X=\xi$ and $Y$ satisfies $\eta(Y)=0$, and (c) $X$ and $Y$ satisfy $\eta(X)=$ $\eta(Y)=0$. The case (a) is trivial. Consider the case (b). We have then $\psi(\xi, Y)=\phi[\xi, Y]-[\xi, \phi Y]$. Since $d \eta=0$, we have $0=d \eta(\xi, Y)=$ $-\eta([\xi, Y])$. This implies that $[\xi, Y]$ is tangent to the leaves of the differential system $\eta=0$. Therefore we get $\psi(\xi, Y)=I[\xi, Y]-[\xi, I Y]$. But $I$ is invariant under $\exp t \xi$; so the right-hand side of the equality is zero. For the case (c), we have

$$
\psi(X, Y)=I[X, Y]-[I X, Y]-[X, I Y]+I[I X, I Y] \text {. }
$$

Since $I_{1}$ is a paracomplex structure on $H_{1}$, the restriction of $I$ to a leaf $H_{t}$ of the differential system $\eta=0$ gives a paracomplex structure on $H_{t}$. Therefore the right-hand side of (1.22) vanishes (cf. [1]).

\section{§2. Principal circle bundles}

Let $\tilde{M}(M, U(1), \pi)$ be a principal circle bundle over a smooth manifold $M$ with projection $\pi$. Suppose that $\tilde{M}$ has a linear connection $\eta$. Then one can find a unique 2-form $\Omega$ on $M$ such that

$$
d \eta=\pi^{*} \Omega .
$$

Proposition 2.1. Under the above circumstances, suppose that $M$ has a paracomplex structure $I$ and that $\Omega$ satisfies

$$
\Omega(I X, Y)+\Omega(X, I Y)=0, \quad X, Y \in \mathfrak{X}(M) .
$$


Then there exist a $(1,1)$-tensor field $\phi$ and a vector field $\xi$ on $\tilde{M}$ such that $(\phi, \xi, \eta)$ is a normal almost paracontact structure on $\tilde{M}$.

Proof. The proof is similar to that of Theorem 6 in [4]. Let $\tilde{X}$ be a tangent vector at $\tilde{p} \in \tilde{M}$, and let us denote by $h \tilde{X}$ (resp. $v \tilde{X}$ ) the horizontal (resp. vertical) component of $X$ with respect to the linear connection. We denote by $X_{\tilde{p}}^{*} \in T_{\tilde{p}}(\tilde{M})$ the lift of a tangent vector $X \in T_{p}(M), p=\pi(\tilde{p})$. Now we define a linear endomorphism $\phi_{\tilde{p}}$ of $T_{\tilde{p}}(\tilde{M})$ by putting

$$
\phi_{\tilde{p}}(\tilde{X})=\left(I \pi_{*} h \tilde{X}\right)_{\tilde{p}}^{*} \text {. }
$$

Then the assignment $\phi: \tilde{p} \rightarrow \phi_{\tilde{p}}$ is a $(1,1)$-tensor field on $\tilde{M}$. The choice of a vertical vector field $\xi$ is quite the same as in [4]. Then $\phi, \xi$ and $\eta$ satisfy the condition (1.1). (1.2) is also proved analogously as in the almost contact case (cf. [4]). $\phi$ leaves horizontal subspaces invariant, and if $\tilde{X}$ is a horizontal vector, then from (2.3) we have $\pi_{*} \phi \tilde{X}=I \pi_{*} \tilde{X}$, which implies (1.3).

Let us denote by $X^{*}$ the horizontal lift of a vector field $X \in \mathfrak{X}(M)$. Then $\phi X^{*}=(I X)^{*}$ holds. So, we have (cf. [4])

$$
\begin{aligned}
\phi\left[X^{*}, Y^{*}\right]=(I[X, Y])^{*}= & ([I X, Y]+[X, I Y]-I[I X, I Y])^{*} \\
= & {\left[\phi X^{*}, Y^{*}\right]-\eta\left(\left[\phi X^{*}, Y^{*}\right]\right) \xi+\left[X^{*}, \phi Y^{*}\right] } \\
& -\eta\left(\left[X^{*}, \phi Y^{*}\right]\right) \xi-\phi\left[\phi X^{*}, \phi Y^{*}\right] .
\end{aligned}
$$

On the other hand, we have

$$
\eta\left(\left[\phi X^{*}, Y^{*}\right]\right)=-d \eta\left(\phi X^{*}, Y^{*}\right)=-\pi^{*} \Omega\left((I X)^{*}, Y^{*}\right)=-\Omega(I X, Y) .
$$

Similarly we have

$$
\eta\left(\left[X^{*}, \phi Y^{*}\right]\right)=-\Omega(X, I Y) .
$$

Substituting (2.5) and (2.6) into (2.4), and taking (2.2) into account, we get $\psi\left(X^{*}, Y^{*}\right)=0$. For the same reason as in [4], we get $\psi\left(X^{*}, \xi\right)=0$. Thus we have $\psi=0$.

Let $\tilde{M}(M, U(1), \pi)$ be a principal circle bundle over a smooth manifold $M$. Let $\eta$ be a linear connection in $\tilde{M}, \xi$ be the fundamental vector field with $\eta(\xi)=1$, and let $\Omega$ be the 2 -form on $M$ satisfying (2.1). Then, as the converse assertion of Proposition 2.1, we have

Proposition 2.2. Given the above notation, suppose that $\tilde{M}$ has a $(1,1)$-tensor field $\phi$ such that $\Sigma=(\phi, \xi, \eta)$ is a normal almost paracontact 
structure. Then $M$ has a paracomplex structure I satisfying (2.2).

Proof. The proof is similar to that of Theorem 1 in Morimoto [5]. Let $X \in T_{p}(M)$ and let us define $I_{p}$ by putting

$$
I_{p} X=\pi_{*} \phi X_{\tilde{p}}^{*},
$$

where $\pi(\tilde{p})=p$ and $X_{\tilde{p}}^{*}$ is the lift of $X$ with respect to $\eta$. By the same reason as in [5], $I_{p}$ is defined and the assignment $I: p \rightarrow I_{p}$ is an almost paracomplex structure on $M$. We have also

$$
(I X)^{*}=\phi X^{*},
$$

where $*$ denotes the horizontal lift. Then, by the same argument as in [5], we have

$$
\begin{aligned}
(I[X, Y])^{*}= & {[I X, Y]^{*}+[X, I Y]^{*}-(I[I X, I Y])^{*} } \\
& +\left\{\eta\left(\left[(I X)^{*}, Y^{*}\right]\right)+\eta\left(\left[X^{*},(I Y)^{*}\right]\right)\right\} \xi .
\end{aligned}
$$

On the other hand, since $\Sigma$ is normal, the almost paracomplex structure on $M \times M$ given in (1.5) has zero torsion tensor. Hence (1.14) is valid. Therefore we have

$$
\begin{aligned}
\eta\left(\left[\phi X^{*}, Y^{*}\right]\right) & =\phi X^{*} \cdot \eta\left(Y^{*}\right)-Y^{*} \cdot \eta\left(\phi X^{*}\right)-\eta\left(\left[\phi^{2} X^{*}, \phi Y^{*}\right]\right) \\
& =-\eta\left(\left[X^{*}-\eta\left(X^{*}\right) \xi, \phi Y^{*}\right]\right)=-\eta\left(\left[X^{*}, \phi Y^{*}\right]\right),
\end{aligned}
$$

which implies that the term enclosed by \{\} in (2.9) is zero. Thus, it follows from (2.9) that $I$ is torsion-free and consequently $I$ is a paracomplex structure. By using (2.10), it is easily seen that $\Omega$ satisfies (2.2).

Definition 2.3. Let $(M, I, g)$ be a parakähler manifold, and let us define a 2 -form $\Omega$ by putting $\Omega(X, Y)=g(X, I Y), X, Y \in \mathfrak{X}(M) ; \Omega$ is closed and is called the fundamental 2-form of $(M, I, \mathrm{~g})$. If $\Omega$ is integral, that is, the cohomology class $[\Omega]$ is an integral class in $H^{2}(M, R)$, then the parakähler metric $g$ is called a parahodge metric, and $(M, I, g)$ is called a parahodge manifold.

ExAmple 2.4. A parahermitian symmetric space $(M, I, g)$ with the 2 nd betti number zero is always parahodge. Let $M$ be the cotangent bundle $T^{*}\left(M_{0}\right)$ over a symmetric $R$-space $M_{0}$. Then $M$ is a parahermitian symmetric coset space of a semisimple Lie group (cf. [1]). The parakähler metric $g$ of $M$ is then induced by the Killing form of the Lie algebra of $G$. If $M_{0}$ is, e.g., $U(n), S O(n), S p(n), U(2 n) / S p(n)$, or the $n$-sphere $(n \geq 2)$, 
then the 2nd betti number of $M$ vanishes; so $g$ is parahodge. Non-trivial examples of parahodge manifolds will be given in the next section.

Theorem 2.5. Let $(M, I, g)$ be a 2n-dimensional parahodge manifold with fundamental 2-form $\Omega$. Suppose that the 1 st betti number $b_{1}(M)$ of $M$ is zero. Then there exist a principal circle bundle $\tilde{M}$ over $M$ and a connection $\eta$ satisfying $d \eta=\pi^{*} \Omega$, where $\pi$ is the projection of $\tilde{M}$ onto $M$. Also $\tilde{M}$ has a normal almost paracontact structure and it has a contact structure.

Proof. Since $\Omega$ is integral, there exists a principal $C^{*}$-bundle $L^{*}$ with connection $\eta$ such that $d \eta=\pi^{*} \Omega$ (cf. Kostant [3]), where $\pi: L^{*} \rightarrow M$ is the projection. Since $b_{1}(M)=0$, the connection $\eta$ is a metric connection (cf. Wallach [8]). Therefore $\eta$ can be considered as a connection in the $U(1)$-subbundle $\tilde{M}$ of $L^{*}$. Hence, by Proposition 2.1, $\tilde{M}$ has a normal almosi paracontact structure. On the other hand, since $\Omega$ is non-degenerate, we have $\eta \wedge(d \eta)^{n}=\eta \wedge \pi^{*} \Omega^{n} \neq 0$ at each point of $\tilde{M}$. Thus $\eta$ is a contact form on $\tilde{M}$.

Remark 2.6. We give here a class of manifolds satisfying the assumptions of Theorem 2.5. Let $(M, I, g)$ be a parahermitian symmetric space with Aut $(M, I, g)$ simple, where $g$ is induced from the Killing form of $G$. Then $(M, I, g)$ is parahodge with $b_{1}(M)=0$ if and only if $M$ is the cotangent bundle over a covering manifold of a symmetric $R$-space $M_{0}^{*}$, which is not the Silov boundary of an irreducible symmetric bounded domain.

\section{§3. Parahodge structures on parahermitian symmetric spaces}

Let $g$ be a real semi-simple Lie algebra, $\mathfrak{h}$ a subalgebra of $\mathfrak{g}$ and let $\sigma$ be an involutive automorphism of $g$ such that $\mathfrak{h}$ is the +1-eigenspace of $\sigma$ in $\mathfrak{g}$. Then the triple $\{\mathfrak{g}, \mathfrak{h}, \sigma\}$ is called a semisimple symmetric triple. We assume that the pair $\{\mathfrak{g}, \mathfrak{h}\}$ is effective. For an effective semisimple symmetric triple $\{\mathfrak{g}, \mathfrak{h}, \sigma\}$, let us consider the following condition (C):

(C) There exists an element $Z \in \mathfrak{g}$ satisfying

i) $\operatorname{ad} Z$ is a semi-simple operator with eigenvalues $0, \pm 1$ only,

ii) $\mathfrak{h}$ coincides with the centralizer of $Z$ in $\mathfrak{g}$.

Note that the condition $(C)$ is equivalent to the condition $\left(\mathrm{C}_{3}\right)$ in [1] (cf. [1]). Now let $\{\mathfrak{g}, \mathfrak{h}, \sigma\}$ be an effective semi-simple symmetric triple satisfying (C), and let $m$ be the -1-eigenspace of $\sigma$ in $\mathfrak{g}$, which is stable under 
ad $Z$. Then we have the decomposition $m=\mathfrak{m}^{+}+\mathfrak{m}^{-}$, where $\mathfrak{m}^{ \pm}$are the \pm 1 -eigenspaces of $\operatorname{ad} Z$ in $\mathrm{g}$.

Remark 3.1. If $\mathrm{g}$ mentioned above has a structure of complex Lie algebra, then $\{\mathfrak{g}, \mathfrak{h}, \sigma\}$ is called an effective complex semi-simple symmetric triple. In this case, $\sigma$ becomes involutive automorphism of $\mathfrak{g}$ regarded as a complex Lie algebra [9]. Furthermore, if $\{\mathfrak{g}, \mathfrak{h}, \sigma\}$ satisfies the condition (C), then the eigenspaces $\mathfrak{h}$ and $\mathfrak{m}^{ \pm}$of ad $Z$ are complex subspaces of $\mathfrak{g}$. But, later on, we will always regard complex semi-simple symmetric triples as real ones, unless otherwise stated.

Now suppose $g$ is real simple. Then we have the following two cases:

Case I (g has no complex Lie algebra structures). In this case the center $z(\mathfrak{h})$ of $\mathfrak{h}$ is one-dimensional and it is generated by $Z$. So we have the decomposition

$$
\mathfrak{h}=[\mathfrak{h}, \mathfrak{h}]+\{\boldsymbol{Z}\}, \quad(\text { direct sum })
$$

where $[\mathfrak{h}, \mathfrak{h}]$ is the commutator subalgebra of $\mathfrak{h}$. Let $G$ be the analytic subgroup generated by $g$ in the simply connected Lie group corresponding to the complexification $\mathfrak{g}^{c}$ of $\mathrm{g}$. Let $C(Z)$ be the centralizer of $Z$ in $G$, and let us denote by $S$ the (closed) analytic subgroup of $G$ generated by [h, $\mathfrak{h}]$.

Case II (g does have a complex Lie algebra structure and consequently, $\mathfrak{g}$ is complex simple). In this case, the center $z(\mathfrak{h})$ of $\mathfrak{h}$ is twodimensional over $R$ and it is generated by $Z$ and $i Z$. We have then the decomposition

$$
\mathfrak{h}=([\mathfrak{h}, \mathfrak{h}]+\{\mathfrak{i} Z\})+\{Z\} . \quad \text { (direct sum) }
$$

Let $G$ be the simply connected (complex) Lie group corresponding to $g$. Then the centralizer $C(Z)$ is connected (cf. [1]). Let $\tilde{S}$ denote the (closed) analytic subgroup of $G$ generated by $[\mathfrak{h}, \mathfrak{h}]+\{i Z\}$.

Then the following proposition is known in Kaneyuki-Kozai [2]:

Proposition 3.2. For Case I, C(Z) has at most two connected components; its identity component $C^{0}(Z)$ is a direct product of the semi-simple derived subgroup $S$ of $C^{0}(Z)$ with the center $\boldsymbol{R}^{+}=\exp t Z$, that is,

$$
C^{0}(Z)=S \cdot R^{+} \quad(\text { direct product })
$$

For Case II, $C(Z)$ is connected and we have 


$$
C(Z)=\tilde{S} \cdot R^{+}, \quad(\text { direct product })
$$

where $\boldsymbol{R}^{+}=\exp t Z$.

Lemma 3.3. Let $B$ be the Killing form of $\mathrm{g}$. Then we have

$$
\begin{aligned}
& B(Z, Z)>0, \\
& B(Z, \mathfrak{m})=B(Z,[\mathfrak{h}, \mathfrak{h}])=0 .
\end{aligned}
$$

Furthermore, if $\mathrm{g}$ is complex semi-simple, then

$$
B(i Z, Z)=0 \text {, }
$$

where $B$ denotes the Killing form of $\mathrm{g}$ regarded as a real Lie algebra.

Proof. We have $B(Z, Z)=\operatorname{Tr}(\operatorname{ad} Z)^{2}=\operatorname{Tr}\left(\left(\operatorname{ad}_{\mathfrak{f}} Z\right)^{2}+\left(\operatorname{ad}_{\mathrm{m}} Z\right)^{2}\right)=$ $\operatorname{Tr}\left(\operatorname{ad}_{\mathfrak{m}} Z\right)^{2}=\operatorname{dim} \mathfrak{m}>0$. On the other hand, we have $B(Z, \mathfrak{m})=0$, since $B(\mathfrak{h}, \mathfrak{m})=0$. Since $\mathfrak{h}$ is the centralizer of $Z$, we get $B(Z,[\mathfrak{h}, \mathfrak{h}])=0$. Suppose that $\mathrm{g}$ is complex. Let us denote by $\tilde{B}$ the Killing form of the complex Lie algebra $g$. Then we obtain $B(i Z, Z)=2 \operatorname{Re} \tilde{B}(i Z, Z)=2 \operatorname{Re} i \tilde{B}(Z, Z)$. From the condition (C) and Remark 3.1, it follows that $\tilde{B}(Z, Z)>0$. Therefore $B(i Z, Z)=0$.

Let us define the linear form $f_{z}$ on $\mathfrak{h}$ by putting

$$
f_{Z}(X)=B(Z, X) \quad X \in \mathfrak{h} .
$$

Then, by Lemma $3.3, f_{z}$ is a nontrivial character of $\mathfrak{h}$. It is known [1] that, in both Case I and Case II, $G / C(Z)$ is a parahermitian symmetric coset space with parakähler metric $g$ induced by the Killing form $B$ of g. Let us consider first Case I. By (3.3), an arbitrary element $a \in C^{0}(Z)$ can be written in the form $a=s r$, where $s \in S, r \in R^{+}$. For Case II, by (3.4), an arbitrary element $a \in C^{0}(Z)=C(Z)$ can be written as $a=\tilde{s} r$, where $\tilde{s} \in \tilde{S}, r \in R^{+}$. Define a map $\chi$ of $C^{0}(Z)$ to the 1 -torus $T^{1}$ in either case by putting

$$
\chi(a)=e^{2 \pi i B(\log r, Z)} .
$$

LEMMA 3.4. $\chi$ is a character of $C^{0}(Z)$ and $\dot{\chi}=2 \pi i f_{Z}$, where $\dot{\chi}$ denotes the differential of $\chi$.

Proof. It is easy to see that $\chi$ is a character of $C^{0}(Z)$. Let us take $X \in \mathfrak{h}$. In Case II, by (3.2), we can write $X=\left(X_{1}+X_{2}\right)+Z_{1}$, where $X_{1} \in[\mathfrak{h}, \mathfrak{h}], X_{2}=\lambda i Z, Z_{1}=\mu Z(\lambda, \mu \in R)$. Then 


$$
\begin{aligned}
\dot{\chi}(X) & =\left.\frac{d}{d t}\right|_{t=0} \chi(\exp t X)=\left.\frac{d}{d t}\right|_{t=0} \chi\left(\exp t\left(X_{1}+X_{2}\right) \cdot \exp t Z_{1}\right) \\
& =\left.\frac{d}{d t}\right|_{t=0} e^{2 \pi i B\left(t Z_{1}, Z\right)}=2 \pi i B\left(Z_{1}, Z\right) \\
& =2 \pi i B\left(X-X_{1}-X_{2}, Z\right) .
\end{aligned}
$$

By Lemma 3.3, we obtain $B\left(X_{1}, Z\right)=B\left(X_{2}, Z\right)=0$. Hence we have $\dot{\chi}(X)$ $=2 \pi i B(X, Z)=2 \pi i f_{Z}(X)$. By the same argument, the lemma is valid for Case I.

LEMMa 3.5. Every almost effective parahermitian symmetric coset space $M$ of a semi-simple Lie group $G$ is a hamiltonian G-space.

Proof. One can express $M$ as a coset space $G / H$, where $H$ is an open subgroup of the centralizer $C(Z)$ in $G$ of an element $Z \in g$ satisfying the condition (C) (cf. [1]). Therefore $G / H$ covers $G / C(Z)$. Let $\tilde{G}$ be the universal covering group of $G$ and $\tilde{C}(Z)$ be the centralizer of $Z$ in $\tilde{G}$. Then we have $G / C(Z)=\tilde{G} / \tilde{C}(Z)$ (cf. [1]). Also we express $M=G / H$ as the coset space $\tilde{G} / \tilde{H}$, where $\tilde{H}$ is the complete inverse image of $H$ in $\tilde{G}$. The coset space $\tilde{G} / \tilde{C}(Z)$ is considered to be the adjoint orbit of $\tilde{G}$ through $Z \in \mathfrak{g}$, which is identified with the co-adjoint orbit of $\tilde{G}$ through $f_{Z} \in \mathrm{g}^{*}$ (= the dual space of $\mathrm{g}$ ) via the Killing form $B$ of $\mathrm{g}$. Therefore $M=G / H$ $=\tilde{G} / \tilde{H}$ is a hamiltonian $\tilde{G}$-space (by Kostant [3]), which implies that $M$ is a hamiltonian $G$-space.

Proposition 3.6. Let $\{\mathfrak{g}, \mathfrak{h}, \sigma\}$ be an effective semi-simple symmetric triple, and $Z \in \mathfrak{g}$ be an element satisfying the condition $(\mathrm{C})$. Let $G / C(Z)$ be a parahermitian symmetric coset space associated with $\{\mathfrak{g}, \mathfrak{h}, \sigma\}$ (cf. Theorem 3.7 in [1]). Let $\Omega$ denote the fundamental 2-form of the parahermitian metric of $G / C(Z)$ induced by the Killing form $B$ of $g$. Then $\Omega$ coincides with the symplectic form constructed by Kostant [3].

Proof. We identify the tangent space $T_{o}(G / C(Z))$ at the origin $o$ in $G / C(Z)$ with $\mathrm{mt}$. Then the paracomplex structure $I_{o}$ of $T_{o}(G / C(Z))$ is identified with $\operatorname{ad}_{\mathrm{m}} Z$ on $\mathrm{m}$ (cf. [1]). Therefore we have $\Omega_{o}(X, Y)=B(X,[Z, Y])$ $=-B([X, Y], Z)=-f_{Z}([X, Y])$, where $X, Y \in \mathfrak{m}$. $f_{Z}$ is considered to be a left-invariant 1 -form on $G$. We have then $-f_{Z}([X, Y])=\left(d f_{Z}\right)(X, Y)$. Let $\pi$ be the projection of $G$ onto $G / C(Z)$. Then, from the above argument, we see that $\pi^{*} \Omega=d f_{Z}$. This implies that our $\Omega$ is the same as the symplectic form constructed by Kostant [3]. 
TheOREM 3.7. Let $\{\mathfrak{g}, \mathfrak{h}, \sigma\}$ be a simple (that is, $\mathfrak{g}$ is simple) symmetric triple satisfying the condition $(\mathrm{C})$. Let $\tilde{G}$ be the simply connected Lie group corresponding to g. Suppose that the centralizer $\tilde{C}(Z)$ in $\tilde{G}$ is connected, or equivalently, $\tilde{G} / \tilde{C}(Z)$ is simply connected. Then the parakähler metric of the parahermitian symmetric coset space $\tilde{G} / \tilde{C}(Z)$ induced by the Killing form of $\mathrm{g}$ is parahodge.

Proof. In Case II, we have $G=\tilde{G}$ and $C(Z)=\tilde{C}(Z)$. Furthermore, since $\tilde{G} / \tilde{C}(Z)$ is simply connected in this case, the assumption that $\tilde{C}(Z)$ is connected is automatically satisfied. So, in view of Lemmas 3.4, 3.5 and Proposition 3.6, a result of Kostant [3] applies and we can conclude that $[\Omega]$ is integral. In Case $I$, there exists a covering homomorphism $\pi$ of $\tilde{G}$ onto $G$. Since $\tilde{G} / \tilde{C}(Z)=G / C(Z)$ is simply connected, we conclude that $\pi(\tilde{C}(Z))=C(Z)$ is connected. Let us consider the character $\chi$ of $C(Z)=C^{0}(Z)$ in Lemma 3.4. The composite map $\tilde{\chi}=\chi \circ \pi$ is the character of $\tilde{C}(Z)$ whose differential is $2 \pi i f_{Z}$. Apply the same result of Kostant [3] as above to $\tilde{\chi}$ to get the assertion of the theorem.

Remark 3.8. A simple parahermitian symmetric coset space $G / C(Z)$ has a structure of the cotangent bundle $T^{*}\left(M_{0}^{*}\right)$ over a symmetric $R$-space $M_{0}^{*}$ (cf. Takeuchi [6]; also see [1]). So $G / C(Z)$ is simply connected if and only if $M_{0}^{*}$ is an irreducible hermitian symmetric space, a quaternionic Grassmannian, $p$-dimensional sphere $(p \geqslant 2), S p(n)$ or the octanion projective plane. Theorem 3.7 has non-trivial meaning only when $M_{0}^{*}$ is hermitian symmetric, that is to say, when and only when $G / C(Z)$ corresponds to one of the pairs $(\mathfrak{I l}(p+q, C), \mathfrak{g l}(p, C)+\mathfrak{g l}(q, C)+C),(\mathfrak{g n}(2 n, C)$, $\mathfrak{g l}(n, C)+C), \quad(\mathfrak{g p}(n+2, C), \quad \mathfrak{g n}(n, C)+C), \quad(\mathfrak{g p}(n, C), \quad \mathfrak{g l}(n, C)+C)$, $\left(E_{6}^{C}, \mathfrak{g} \mathfrak{0}(10, C)+C\right)$ or $\left(E_{7}^{C}, E_{6}^{C}+C\right)$; see [1]. Because, if $M_{0}^{*}$ is the one above other than a hermitian symmetric space, then the $2^{\text {nd }}$ betti number $b_{2}(G / C(Z))=b_{2}\left(M_{0}^{*}\right)$ vanishes (see [7]). So [ $\left.\Omega\right]$ is trivially integral in this case. Thus Theorem 3.7 leads to:

Corollary 3.9. Suppose $G$ is simple. Then the simply connected coset spaces $G / C(Z)$ with a parahodge structure induced by the Killing form are given up to infinitesimal equivalence by the pairs $(\mathfrak{g}, \mathfrak{h})$ (in the symmetric triple $(\mathfrak{g}, \mathfrak{h}, \sigma))$ as follows: Those with $b_{2}=0$ are $\left(\mathfrak{B} \mathfrak{u}^{*}(2 p+2 q), \mathfrak{S} \mathfrak{u}^{*}(2 p)+\right.$

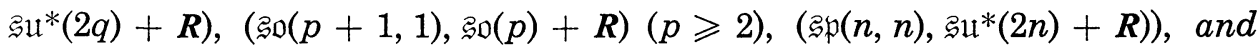
$\left(E_{6}^{4}, \mathfrak{g} \mathfrak{O}(1,9)+R\right)$. Those with $b_{2} \neq 0$ are $(\mathfrak{g l}(p+q, C), \mathfrak{g l}(p, C)+\operatorname{gll}(q, C)$

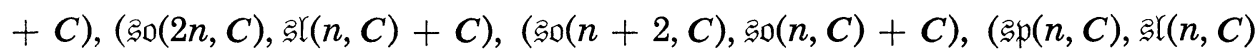


$+C),\left(E_{6}^{C}, \mathfrak{g} \mathfrak{O}(10, C)+C\right)$, and $\left(E_{7}^{C}, E_{6}^{C}+C\right)$.

We shall now see when we can replace the assumption in Theorem 3.7 by a weaker one. Let $G$ be a simple Lie group satisfying the same assumption put before Proposition 3.2. Suppose that $C(Z)$ is not connected. Then, by Proposition 3.2, we can write $C(Z)$ as

$$
C(Z)=C^{0}(Z) \cup x_{0} C^{0}(Z) \quad \text { (disjoint), }
$$

where $x_{0} \in C(Z)$ satisfies $x_{0}^{2} \in C^{0}(Z)$.

Lemma 3.10. Let $\chi$ be a character of $C^{0}(Z)$. Suppose $x_{0}^{2}=e$. Then $\chi$ extends to a character of $C(Z)$ if and only if

$$
\chi\left(x_{0} c x_{0}\right)=\chi(c)
$$

holds for every $c \in C^{0}(Z)$.

Proof. Let $H$ be the cyclic subgroup generated by $x_{0}$. Then we have the local direct product decomposition $C(Z)=C^{0}(Z) \cdot H$. Take an element $c \in C(Z)$ and put $c=c_{0} h, c_{0} \in C^{0}(Z), h \in H$. Let us define $\tilde{\chi}(c)=\chi\left(c_{0}\right)$. Then $\tilde{\chi}$ is the desired extension of $\chi$.

TheOREm 3.11. Let $\{\mathfrak{g}, \mathfrak{h}, \sigma\}$ be a simple symmetric triple satisfying the condition (C). Let $G$ be the analytic subgroup generated by $g$ in the simply connected Lie group corresponding to the complexification of $\mathfrak{g}$, and let $C(Z)$ be the centralizer of $Z$ in $G$. Let $\chi$ be the character of $C^{0}(Z)$ whose differential is $2 \pi i f_{z}$. Suppose that there exists $x_{0} \in C(Z)$ satisfying $x_{0}^{2}=e$, (3.10) and (3.11). Then the parakähler metric of parahermitian symmetric space $G / C(Z)$ induced by the Killing form of $\mathrm{g}$ is parahodge.

Proof. By Lemma 3.10, $\chi$ extends to a character of $C(Z)$, which is also denoted by $\chi$. Let $\tilde{G}$ be the universal covering group of $G$, and $\tilde{C}(Z)$ be the centralizer of $Z$ in $\tilde{G}$. Then, as is remarked in the proof of Lemma 3.5 , we have $G / C(Z)=\tilde{G} / \tilde{C}(Z)$. Let us denote by $\pi$ the projection of $\tilde{G}$ onto $G$. Then, we have $\pi(\tilde{C}(Z))=C(Z)$. The composite map $\tilde{\chi}=\chi_{\circ} \pi$ is the character of $\tilde{C}(Z)$ whose differential is $2 \pi i f_{Z}$. In view of Lemma 3.5 , a result of Kostant [3] applies and we can conclude that $[\Omega]$ is integral.

We will give a sufficient condition for the character $\chi$ of $C^{0}(Z)$ to extend to a character of $C(Z)$.

Proposition 3.12. Let $x_{0}$ be an element satisfying (3.10). Suppose $x_{0}^{2}=e$, and suppose that $x_{0}$ normalizes $S$ and $\boldsymbol{R}^{+}$, where $S$ and $\boldsymbol{R}^{+}$are the 
same as in Proposition 3.2, Case I. Then the character $\chi$ of $C^{0}(Z)$ whose differential is $2 \pi i_{Z}$ satisfies (3.11); in other words, $\chi$ extends to a character of $C(Z)$.

Proof. Let us write $c \in C^{0}(Z)$ in the form $c=s r$, where $s \in S$ and $r \in \boldsymbol{R}^{+}$. Then we have $x_{0} c x_{0}=x_{0} s x_{0} \cdot x_{0} r x_{0}$ and so $\chi\left(x_{0} c x_{0}\right)=e^{2 \pi i B\left(Z, \log x_{0} r x_{0}^{-1}\right)}$. We write $r=\exp y$; then we see $x_{0} r x_{0}^{-1}=\exp \left(\left(\operatorname{Ad} x_{0}\right) y\right)$. Hence

$$
\begin{aligned}
\chi\left(x_{0} c x_{0}\right) & =e^{2 \pi i B\left(Z, \operatorname{Ad}\left(x_{0}\right) \log r\right)}=e^{2 \pi i B\left(\operatorname{Ad}\left(x_{0}^{-1}\right) Z, \log r\right)} \\
& =e^{2 \pi i B(Z, \log r)}=\chi(c) .
\end{aligned}
$$

ExAmple 3.13. Let $G=S L(p+q, R)$, and let $Z=\operatorname{diag}\left((-q /(p+q)) E_{p}\right.$, $\left.(p /(p+q)) E_{q}\right)$. Then we have

$$
\begin{aligned}
C(Z) & =\left[\left(\begin{array}{cc}
A & 0 \\
0 & B
\end{array}\right) \in S L(p+q, R)\right]=S(G L(p, R) \times G L(q, R)), \\
C^{0}(Z) & =\left[\left(\begin{array}{cc}
A & 0 \\
0 & B
\end{array}\right) \in C(Z) ; \operatorname{det} A>0\right] \\
\boldsymbol{R}^{+} & =\exp t Z=\operatorname{diag}\left[e^{-(q / p+q) t} E_{p}, e^{(p / p+q) t} E_{q}\right] \\
S & =\left[\left(\begin{array}{cc}
A & 0 \\
0 & B
\end{array}\right) \in C(Z): \operatorname{det} A=\operatorname{det} B=1\right] .
\end{aligned}
$$

Choose $x_{0}$ to be

$$
x_{0}=\operatorname{diag}\left(-1,1, \stackrel{p-1}{\cdots}, 1,-1,1, \frac{q-1}{\cdots}, 1\right) .
$$

Then $x_{0}$ normalizes $S$ and $\boldsymbol{R}^{+}$. Therefore the metric on $S L(p+q, \boldsymbol{R}) /$ $S(G L(p, R) \times G L(q, R))$ induced by the Killing form is parahodge.

\section{BIBLIOGRAPHY}

[1] S. Kaneyuki and M. Kozai, Paracomplex structures and affine symmetric spaces, Tokyo J. Math., 8 (1985), 301-318.

[2] S. Kaneyuki and M. Kozai, On the isotropy subgroup of the automorphism group of a parahermitian symmetric space, to appear in Tokyo J. Math.

[3] B. Kostant, Quantization and unitary representations, Lecture Notes in Mathematics, vol. 170, Springer-Verlag, Berlin, 87-208.

[4] A. Morimoto, On normal almost contact structures, J. Math. Soc. Japan, 15 (1963), $420-436$.

[5] A. Morimoto, On normal almost contact structures with regularity, Tohoku Math. J., 16 (1964), 90-104.

[6] M. Takeuchi, Cell decompositions and Morse equalities on certain symmetric spaces, J. Fac. Sci. Univ. Tokyo. Section I, 12 (1965), 81-192.

[7] M. Takeuchi, Stability of certain minimal submanifolds of compact Hermitian symmetric spaces, Tohoku Math. J., 36 (1984), 293-314. 
[8] N. R. Wallach, Symplectic Geometry and Fourier Analysis; Math. Sci. Press, Mass. 1977.

[ 9 ] S. S. Koh, On affine symmetric spaces, Trans. Amer. Math. Soc., 119 (1965), 291-309.

Soji Kaneyuki

Sophia University, Tokyo

Kioicho, Chiyoda-ku

Tokyo 102, Japan

and

University of Massachusetts/Amherst

Floyd L. Williams

University of Massachusetts/Amherst

Ma. 01003 U.S.A. 